\title{
Endoscopic ultrasonography-guided liver abscess drainage using a dedicated, wide, fully covered self-expandable metallic stent with flared-ends
}
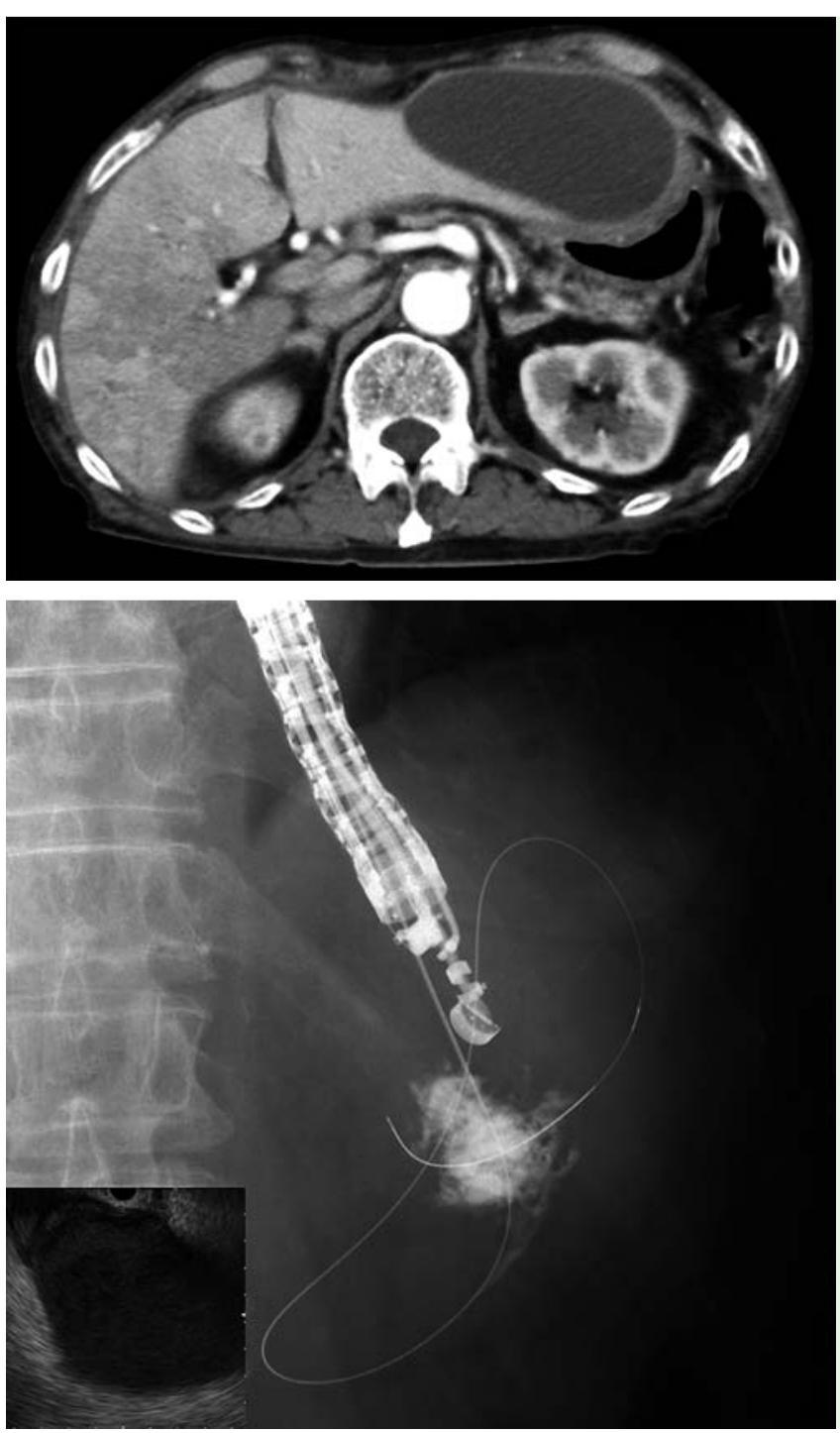

Endoscopic ultrasonography (EUS)-guided drainage of liver abscesses has recently become available. We present here a case of successful drainage of a liver abscess using a dedicated wide fully covered selfexpandable metallic stent (FCSEMS) with flared ends.

An 84-year-old man was admitted to another hospital with a history of high fever and epigastric pain for 12 days. Computed tomography (CT) revealed a $10.3 \times$ $6.1-\mathrm{cm}$ abscess in the left lobe of the liver ( Fig. 1). He was referred to our hospital
Fig. 1 An abscess in the left lobe of the liver identified at computed tomography (CT) in an 84-year-old man with a 12-day history of high fever and epigastric pain.

Fig. 3 Radiograph showing placement of the guidewire into the cavity of the liver abscess. Inset: endoscopic ultrasonography (EUS) image showing the liver abscess as a heterogeneous hypoechoic lesion in the gastrohepatic space.

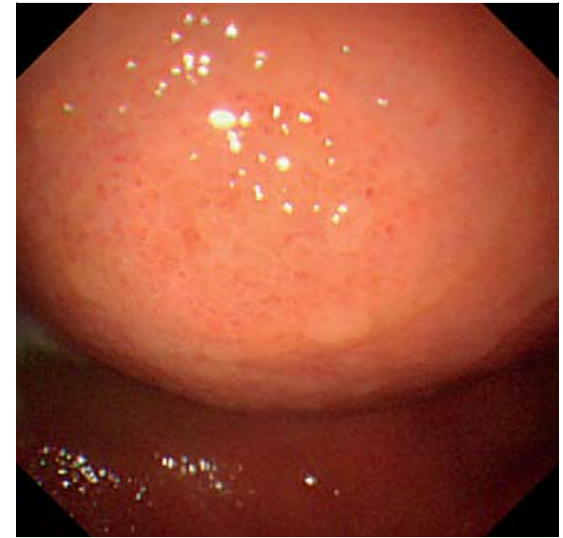

Fig.2 Esophagogastroduodenoscopy (EGD) shows a bulging mass in the upper body of the stomach

ends (NAGI stent, $16 \times 3 \mathrm{~cm}$; TaewoongMedical, Seoul, Korea) was placed, without any complications ( $\bullet$ Fig. 4, $\bullet$ Fig.5). After stone clearance from the common bile duct, the patient was discharged on postoperative day 10 without removal of the SEMS ( $\bullet$ Fig. 6).

Seven cases of EUS-guided drainage of liver abscess, including one case of multiple abscesses, have been reported to date $[1,2]$. The left lobe of the liver, the caudate lobe, and the gastrohepatic space usually lie in close proximity to the stomach or duodenum [1]. Therefore, EUS-guided liver abscess drainage might be safe and effective in the management of these areas. Single or double plastic stents were used in most of the reported cases [1]; the newly designed dedicated anchoring FCSEMS with a "yo-yo" shape was placed in only one patient [2]. It has been suggested that a dedicated FCSEMS is the ideal stent for treating liver abscesses and pancreatic fluid collection because of its antimigration feature and because it allows direct insertion of an endoscope through it [3].

Endoscopy_UCTN_Code_TTT_1AS_2AC

Competing interests: None 



Fig.4 The dedicated, wide, fully covered selfexpandable metallic stent (FCSEMS) with flared ends (NAGI stent; Taewoong-Medical, Seoul, Korea)
Fig. 5 Radiograph showing placement of the NAGI stent into the cavity of the liver abscess. Inset: endoscopic view of the purpose-designed stent.

\section{Hiroshi Kawakami ${ }^{1}$, Kazumichi \\ Kawakubo², Masaki Kuwatani ${ }^{3}$, Yoshimasa Kubota ${ }^{1}$, Yoko Abe ${ }^{1}$, Shuhei Kawahata', Kimitoshi Kubo', Naoya Sakamoto ${ }^{2}$}

${ }^{1}$ Department of Gastroenterology and Hepatology, Hokkaido University Hospital 2 Department of Gastroenterology and Hepatology, Hokkaido University Graduate School of Medicine, Sapporo, Japan ${ }^{3}$ Division of Endoscopy, Hokkaido University Hospital

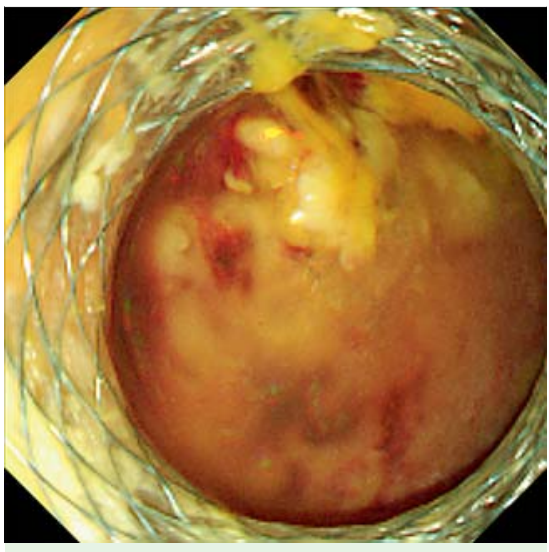

Fig. 6 Endoscopic view through the NAGI stent on postoperative day 8 , showing only necrotic tissues on the surface of the liver.
Bibliography

DOI http://dx.doi.org/

10.1055/s-0034-1391125

Endoscopy 2014; 46: E982-E983

(c) Georg Thieme Verlag KG

Stuttgart · New York

ISSN 0013-726X

2 Alcaide N, Vargas-Garcia AL, de la SernaHiguera $C$ et al. EUS-guided drainage of liver abscess by using a lumen-apposing metal stent (with video). Gastrointest Endosc 2013; 78: 941 - 942

3 Kawakami H, Itoi T, Sakamoto N. Endoscopic ultrasound-guided transluminal drainage for peripancreatic fluid collections: Where we are now? Gut Liver 2014; 8: 341 -355
Corresponding author

\section{Hiroshi Kawakami, MD, PhD}

Department of Gastroenterology and Hepatology Hokkaido University Hospital

Kita 14, Nishi 5, Kita-ku

Sapporo 060-8648

Japan

Fax: +81-11-7067867

hiropon@med.hokudai.ac.jp 